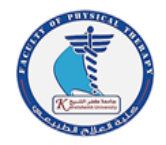

\title{
Effect of Body Mass Index on Craniovertebral Angle and Shoulder Angle in Egyptian Adolescents
}

\author{
Somaya NM Elsayed ${ }^{1 *}$, Haytham M Elhafez ${ }^{2}$, Mayada A Mahmoud ${ }^{3}$ \\ ${ }^{1}$ BSc. Physical Therapy, Basic Science Department, Faculty of Physical Therapy, Cairo University, Egypt. \\ ${ }^{2}$ Professor of Physical Therapy, Basic Science Department, Faculty of Physical Therapy, Cairo University; \\ Dean of Faculty of Physical Therapy, Suez University, Egypt. \\ ${ }^{3}$ Lecturer of Physical Therapy, Basic Science Department, Faculty of Physical Therapy, Cairo University, Egypt.
}

\author{
*Correspondence to \\ Somaya NM Elsayed \\ Basic Science \\ Department Faculty of \\ Physical Therapy, Cairo \\ University, Egypt. \\ Tel: 01115593279 \\ Email: \\ somyaowais@gmail.com
}

\author{
Published online: \\ March 2020
}

\begin{abstract}
:
Purpose: To explore the effect of body mass index (BMI) on Craniovertebral Angle (CVA) and Shoulder Angle (SA) in Egyptian Adolescents.

Methods: Cross Sectional Study designe was used. Eighty-six physical therapy students were selected randomly, and their age were ranged from 18-27 years. They were divided into three groups according to BMI. The first group with normal BMI $\left(18.5-24.9 \mathrm{Kg} / \mathrm{m}^{2}\right.$ ), while the second group was overweight (BMI was ranged from 25$29.9 \mathrm{Kg} / \mathrm{m}^{2}$ ). The third group was obesity class I (BMI was ranged from 30-34.9 $\mathrm{Kg} / \mathrm{m}^{2}$ ). Photogrammetry method used (the photographic sessions by mobile phone Samsung J-7 Camera and use of Corel Draw software to analyze the pictures).

Results: A significant difference was detected between normal BMI and overweight $(p<0.02)$ and significant difference between normal BMI and obese $(p<0.03)$. For SA there was significant difference between normal BMI and overweight $(\mathrm{p}<0.02)$. However, for CVA the relation between overweight and obese was not significant $(\mathrm{p}>0.2)$, and for SA non-significant difference between normal BMI and obese $(p>0.41)$ and non-significant difference between overweight and obese $(p>0.2)$.

Conclusion: overweight and obesity change CVA and forward head posture and overweight changed SA and so rounded shoulder appeared. However, obesity did not change SA and rounded shoulder.
\end{abstract}

Keywords: Body Mass Index, Craniovertebral angle, Shoulder angle.

\section{Introduction}

Obesity is defined as storage of excessive body fat and lead to major health problem, increase morbidity, mortality rate and increase diseases such as cardiovascular diseases, respiratory diseases, musculoskeletal problems as osteoarthritis. (1) Posture is position of body parts in relation to each other parts. Inadequate posture leads to shifting of center of gravity (COG) from proper alignment, leading to more compensation by other body part. Obesity shifts COG from normal position lead to change moment arm and increase stress on joint and change its functional angle.(2) The head is rounded with a shoulder shape and has a significant postural abnormality and is associated with cervical and migraine headaches, fascial tightness, irregular movement of the scapular and even temporomandibular disturbances.(3) Potential head postures are associated with shorter head spine, splenius and semispinalis capitis and cervicis, cervical spinae erector and levator scapulae.(4)

Prolonged malalignment of head from normal alignment leads to forward head posture. Failure of the head to align with the vertical axis of the body leads to more misalignments such as rounded shoulder posture (RSP). (5) Postural abnormality such rounded shoulder posture is malalignment of shoulder from normal alignment. It is caused by muscle imbalance between shorted pectoralis major, pectoralis minor, levator 
scapula and stretch weakness of middle trapezius muscle and rhomboids.(4) Kwon et al., concluded that different head positions affect posture of all body part.(6) Forward head posture (FHP), which induces a round shoulder posture as a result of muscular disequilibrium, contributes to changes in the spinal curvature and increases tension on various body parts.(7) Much work into the impact of the Body Mass Index on FHP and rounded shoulder (RS) remains incomplete, although several studies have been performed to determine the relationship between FHP and cervical pain or chest kyphosis or temporomandibular joint. This study was designed to investigate the effect of BMI on craniovertebral angle (forward head posture) and on shoulder angle (rounded shoulder posture) in asymptomatic subjects.

\section{Subjects and Methods}

\subsection{Design}

This study was cross sectional study. The Research Ethical Committee of the Faculty of Physical Therapy, Cairo University gave an acceptance for the study. The study was conducted between September 2019 and December 2019. The study had followed the Guidelines of the Declaration of Helsinki on the conduct of human research.

\subsection{Randomization}

Each participant signed a consent form after having clarified the existence, intent and advantages of the experiment, notified them of their right to reject or withdraw at any time, and the confidentiality of any data obtained. Upon randomization for two hundred students (by distribution of sealed envelopes), participants are selected. Participants were assigned into three groups.

\subsection{Participants}

A sample of eighty-six physical therapy students was recruited from the Faculty of Physical Therapy, Cairo University, Egypt. The participants were chosen and divided into three groups according to their BMI. Group "A" involved 33 subjects with their BMI was ranged from 18.5-24.9 kg/m2 and Group "B" involved 28 subjects (Over Weight) with their BMI was ranged from $25-29.9 \mathrm{Kg} / \mathrm{m} 2$ and Group "C" included 25 obese subjects (Class I) with their BMI was ranged from 30$34.9 \mathrm{Kg} / \mathrm{m} 2$. Their ages were ranged from $18-27$ years old, and their BMI did not exceed $35 \mathrm{Kg} / \mathrm{m} 2$. The participants were excluded if they had neck pain, shoulder pain, recent shoulder fracture, cervical disc prolapse, radiculopathy, and subjects had musculoskeletal disorders or leg length discrepancy, history of neurological disorder affecting lower extremities. In addition, subjects had symptoms of vertigo, dizziness, visual or auditory problems, an autoimmune disease or systemic disease were excluded.

\subsection{Assessment}

Assessment of CVA and SA were applied by Corel Draw Graphics Suit "x7 software" and mobile phone Samsung J7 Camera. It is valid and reliable tool for assessment of CVA and SA. (8) Subjects were given the intent and procedure of the study in verbal instructions. Subjects were instructed to uncover from the head to the thoracic spine. Then they stood in a relaxed position at the marker on the floor, were making cervical flexion and extension to be relaxed. Three images would be taken for each subject and these images were uploaded on the software to be analyzed according to the following two main parts: First part; Measurement of craniovertebral angle: CVA determine forward head posture and it is calculated by taking a line from $\mathrm{C} 7$ to tragus of the ear and horizontal line from C7. Second part; Measurement of Shoulder angle: SA determines rounded shoulder posture. It was calculated by taking a line from anterolateral aspect of acromion to $\mathrm{C} 7$ and a horizontal line from anterolateral aspect of acromion.

\subsection{Outcome Measures}

If CVA is $<50$, it is considered as forward head posture. If $\mathrm{SA}$ is $<52$, it is considered as rounded shoulder posture

\section{Statistical analysis}

The total sample size ( 86 subjects) was calculated at $\alpha=0.05$. Results are expressed as mean \pm standard deviation. The current test involved one independent variable was the body mass index; which had 3 levels (normal, overweight, and obese) and two dependent variables which are craniovertebral angle and shoulder angle. For data analysis, the computer program Statistical Social Science Package (SPSS) (19 windows version) has been used. The $\mathrm{p}$ value was considered $<0.05$ and $<0.01$ considered quite significant.

\section{Results}

For CVA classes compartments statistically significant between normal BMI / overweight difference $(p<0.02)$ and statistically significant between normal BMI / obese difference $(\mathrm{p}<0.03)$ were observed. Nevertheless, there was statistically significant considerable difference between overweight and obese $(p>0.2)$ for SA between normal BMI and overweight, $(\mathrm{p}<0.02)$ and between normal BMI and Obese $(p>0.41)$ and not statistically significant between overweight and obese ( $>0.2)$. Overweight and obesity are not considerably different.

Table (1): Demographic data of the studied subjects

\begin{tabular}{cccccc}
\hline & $\begin{array}{c}\text { Number of } \\
\text { subjects }\end{array}$ & $\begin{array}{c}\text { Age } \\
\text { "Years" }\end{array}$ & $\begin{array}{c}\text { Weight } \\
\text { "Kg" }\end{array}$ & $\begin{array}{c}\text { Height } \\
\text { "m" }\end{array}$ & $\begin{array}{c}\text { BMI } \\
\text { "Ratio" }\end{array}$ \\
\hline Normal & \multirow{2}{*}{33} & 19.41 & 57.30 & 1.61 & 21.98 \\
Weight & & \pm 1.03 & \pm 6.65 & \pm 0.06 & \pm 1.71 \\
Over & 28 & 19.21 & 70.25 & 1.61 & 26.78 \\
Weight & \multirow{2}{*}{25} & \pm 0.42 & \pm 7.23 & \pm 0.07 & \pm 1.44 \\
Obese & 19.04 & 86.8 & 1.58 & 32.7 \\
\hline BMI: Body Mass Index, Kg: Kilogram, M: meter & &
\end{tabular}

BMI: Body Mass Index, Kg: Kilogram, M: meter 
Table (2): The mean and standard deviation of CVA and SA in degrees of the studied subjects

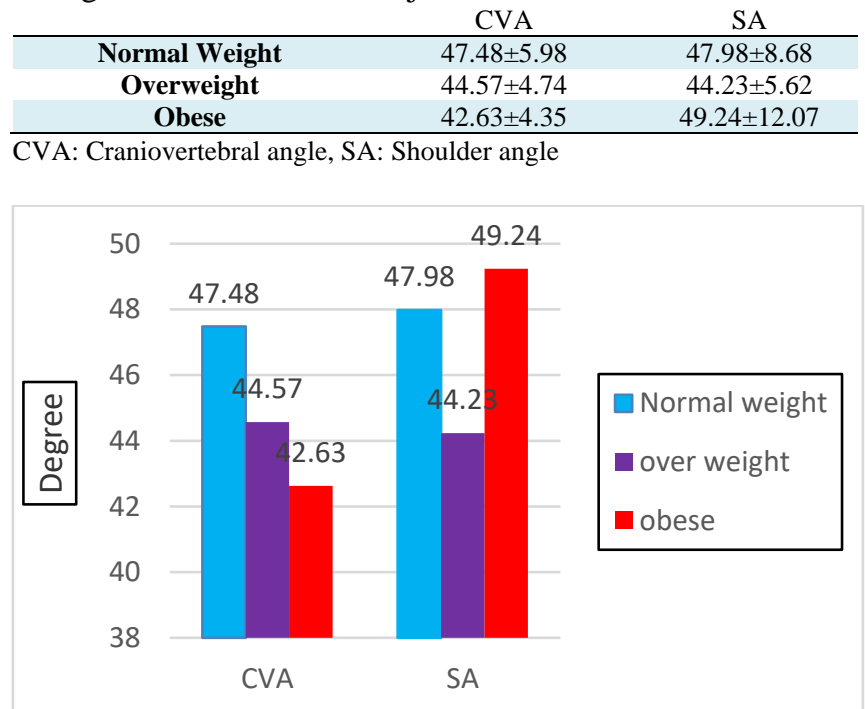

Fig (1): Mean of CVA and SA of studied subjects

\section{Discussion}

The findings had shown that there was no substantial CVA variation from overweight to obese, and no significant difference in SA from normal BMI and obese to overweight and obese. For CVA, however, there were considerable variations between normal BMI and overweight and between normal BMI and obese and for SA, between normal BMI and overweight.

Standing position was only used because in sitting position the probability of postural abnormality in cervical spine and rounded shoulder might be compensated. As well this would lead to change of muscle fiber length in cervical spine, and cause inability to maintain upright sitting posture. (6)

In the study of Kim and Kim, their results showed no significant correlation was detected in standing and sitting position in CVA and RSP.(7) The limit of FHP was determined as $<50^{\circ}$.(3)

Kim and Kim were assessed rounded shoulder posture by height of acromion of dominant shoulder.(7) Photography was utilized in this study to determine the shoulder angle rather than the supine position, to test the misalignments of corporal segments by photographic measurements of the sagittal postures of cervical spine and the shoulder. The photographic measurement of sagittal postures in the cervical spine and the shoulder is being increased in order to study misalignments on body parts, with several studies confirming that photogrammetry is highly reliable. (2)

Although the supine position is accurate in assessing symptoms for the rounded shoulder posture (RSP), the longitude of the pectoral minor muscle is typically measured. The height of the acromion of the dominant shoulder joint of only one hand was determined in the RSP gage and, as measured in the supine position, there was a constraint in the estimation of the deformity of the spinal fold and the spatial adjustment of the scapula due to FHP. FHP is reduced as determined by the supine angle.

Alternation of body posture change position of head leads to forward head posture (FHP). FHP leads to RSP. RSP leads to increasing of thoracic kyphosis. (5) Kwon et al. concluded that the different head positions affect the posture of your body and thus affect your muscle activity. Future head posture rounded shoulder (FHPR) postural changes can affect the scapular position and the joints of the shoulder. (6)

Thigpen et al. concluded that during the loading of the forward flexion test, greater anterior scapular inclination and internal rotation and decreased upward scapular rotation were shown in individuals free from FHRSP shoulder pain with a corresponding lower serratus level and lower scapular internal rotations with comparable lower level of anterior muscle activity.

This supports the clinical hypothesis of the potential risk that alters postural changes linked to the FHRSP during overhead tasks. (9) FHP is one of the most common cervical abnormalities that predispose people to disease. In a lower CVA, more FHP is shown. Higher CVAs were available in the study of smartphones over 4 hours a day. Another study revealed that many users of smartphones have increased cervical angle pain in the neck. (10)

Neck musculoskeletal disorder and cervical dysfunction lead to forward head posture, thoracic kyphosis and rounded shoulder posture. These were caused by imbalance of pectoralis major, pectoralis minor, posterior neck muscles, and middle fibers of trapezius muscle. (11)

Saaid et al claimed that FHP is moderate and moderately altered by a rounded shoulder posture. The rounded location of the shoulder did not change however by the extent of the stance of the front head. (12)

The significant decrease in CVA between normal weight (Normal BMI) and overweight, between normal weight and obese is due to affection of posture by obesity. This leads to shifting of COG anteriorly, and consequently affects torque (Torque $=$ Force $\mathrm{x}$ Moment Arm). Affection of torque will lead to postural changes to compensate this abnormality as well; will decrease craniovertebral angle and forward head posture.

Although the current study reveals objective data with statistically significant differences, but there are some limitations. The main limitation is the small sample size so, larger studies are necessary to confirm these findings. 


\section{Conclusion}

It was concluded that, there was change in CVA between normal BMI and overweight and between normal BMI and obese. There was change in SA between normal BMI and overweight. However, there were no change in CVA between overweight and obese and no change in SA between normal BMI and obese and between overweight and obese.

Funding: Not funded.

\section{Conflict of Interests}

Authors declare no potential conflicts of interest.

\section{Acknowledgments}

We would like to thank all individuals who contributed to the completion of this work, especially participants.

\section{References}

1. Ibrahim A, Mosaad D, Ibrahim O. "Influence of Body Mass Index on Lumbar Proprioception in Adult Subject". M.Sc. Thesis Faculty of Phys Ther. Cairo Univ. 2018.

2. Ruivo RM, Pezarat-Correia P and Cartia AI. "Cervical and shoulder postural assessment of adolescents between 15 and 17 years old and association with upper quadrant pain". Braz J Phys Ther. 2014; 18 (4): 364-371.

3. Diab AA and Moustafa IM. "The efficacy of forward head correction on nerve root function and pain in cervical spondylotic radiculopathy: a randomized trial". Clin Rehabil. 2011; 26 (4): 351361.

4. Lynch SS, Thigpen CA, Mihalik JP, Prentice WE, Padua D. "The Effects of an Exercise Intervention on Forward Head and Rounded Shoulder Postures in Elite Swimmers". Br J Sports Med. 2010; 44:376-381.

5. Singla D and Veqar Z. "Association between Forward Head, Rounded Shoulders, and Increased Thoracic Kyphosis: A Review of the Literature". J Chiropr Med. 2017; 16 (3): 220-229.

6. Kwon JW, Son SM, Lee NK. "Changes in upperextremity muscle activities due to head position in subjects with a forward head posture and rounded shoulders". J. Phys. Ther. Sci. 2015; 27 (6):17391742.

7. Kim EK, Kim JS. "Correlation between Rounded Shoulder Posture, Neck Disability Indices and Degree of Forward Head Posture". J Phys Ther Sci. 2016; 28 (10): 2929-2932.

8. Saad KR, Colombo AS, Ribeiro AP, Joao SM. "Reliability of Photogrammetry in the Evaluation of the Postural Aspects of Individuals with Structural Scoliosis". J Bodyw Mov Ther. 2012; 16 (2): 210-216.

9. Thigpen CA, Padua DA, Michener LA, Guskiewicz K, Giuliani C, Keener JD, Stergiou N. "Head and
Shoulder Posture Affect Scapular Mechanics and Muscle Activity in Overhead Tasks". J Electromyogr Kinesiol. 2010; 20 (4): 701-709.

10. Jung SI, Lee NK, Kang KW, Kim K, Lee DY "The Effect of Smartphone Usage Time on Posture and Respiratory Function". J. Phys. Ther. Sci. 2016; 28: 186-196.

11. Park HC, Kim YS, Seok SH, Lee SK. "The Effect of Complex Training on the Children with all of the Deformities Including Forward Head, Rounded Shoulder Posture, and Lumbar Lordosis". J. Exerc. Rehabil. 2014; 10 (3): 172-175.

12. Saaid AA, Mohammed GA, Elhafez HM. "Correlation between Degree of Forward Head Posture and Rounded Shoulder Posture in Physical Therapy Students". Med. J. Cairo Univ. 2019; 87 (1): 511-515. 